\title{
A Novel Complementary Method for the Point-Scan Nondestructive Tests Based on Lamb Waves
}

\author{
Rahim Gorgin, Zhanjun Wu, and Yuebin Zheng \\ State Key Laboratory of Structural Analysis for Industry Equipment, School of Aeronautics and Astronautics, \\ Dalian University of Technology, Dalian, Liaoning 116024, China
}

Correspondence should be addressed to Zhanjun Wu; wuzhj@dlut.edu.cn

Received 6 July 2014; Accepted 10 November 2014; Published 25 November 2014

Academic Editor: R. Ganguli

Copyright (C) 2014 Rahim Gorgin et al. This is an open access article distributed under the Creative Commons Attribution License, which permits unrestricted use, distribution, and reproduction in any medium, provided the original work is properly cited.

\begin{abstract}
This study presents a novel area-scan damage identification method based on Lamb waves which can be used as a complementary method for point-scan nondestructive techniques. The proposed technique is able to identify the most probable locations of damages prior to point-scan test which lead to decreasing the time and cost of inspection. The test-piece surface was partitioned with some smaller areas and the damage probability presence of each area was evaluated. $A_{0}$ mode of Lamb wave was generated and collected using a mobile handmade transducer set at each area. Subsequently, a damage presence probability index (DPPI) based on the energy of captured responses was defined for each area. The area with the highest DPPI value highlights the most probable locations of damages in test-piece. Point-scan nondestructive methods can then be used once these areas are found to identify the damage in detail. The approach was validated by predicting the most probable locations of representative damages including through-thickness hole and crack in aluminum plates. The obtained experimental results demonstrated the high potential of developed method in defining the most probable locations of damages in structures.
\end{abstract}

\section{Introduction}

Nondestructive inspection of structures for the identification of hidden defects that may occur due to abrupt impact or sequential accumulation of defect under fatigue loading during their operation has always been of great industrial interest. The flaws are a real threat for the reliability of a structure, as they can rapidly grow to cause catastrophic failure of the whole system. Hence, a significant amount of work has been conducted in order to determine the location and size of defects in structures which is vital for further investigation about remaining life or residual strength of the damaged structure. This work is always done in parallel with the development of nondestructive techniques [1]. Much effort is being taken to find the most reliable NDE methods for this task.

Point-scan nondestructive tests such as ultrasonic provide diagnostic image of the test-piece which highlights the damage in detail [2]. However, the need to scan the specimen in two dimensions makes ultrasonic inspection a time consuming procedure.
For excellent propagation capability even in materials with high attenuation ratio (e.g., $\mathrm{CF} / \mathrm{EP}$ composites) and very high sensitivity to structural damage, Lamb waves recently have been examined as a means of establishing novel NDE tools [3]. When Lamb wave is generated from an actuator and incident with damage, scattering happens in all directions. Such scattering waves collected by sensors can be utilized to investigate the damage. In this way, Lamb waves can provide an area-scan inspection of the test-piece which can be used as a complement method for point-scan nondestructive test to minimize the time of inspection.

Lamb waves were first described in theory by Lamb in 1917 [4]. They refer to elastic waves that are generated in a solid plate with free boundaries for which the displacements correspond to different basic propagation modes: symmetric and antisymmetric. It was long after Horace Lamb theory about the existence of Lamb waves that for the first time Firestone and Ling 1951 succeeded in experimentally generating Lamb waves in plates [5]. Nowadays, Lamb waves can be actively generated and collected with various methods such as ultrasonic probes coupled with angle-adjustable Perspex wedges 
[6]; Hertzian contact transducer [7]; laser-based ultrasonic (LBU) for Lamb wave generation and laser interferometer for their acquisition [8]; piezoelectric lead zirconate titanate (PZT) elements [9]; and interdigital transducers such as polyvinylidene fluoride (PVDF) piezoelectric polymer film [10].

Worlton [11] was the first to demonstrate several applications of Lamb waves in NDT. He has illustrated the ability of Lamb waves to identify many types of flaws lying close to the surface of a metal submerged in water. Later on Lehfeldt and Höller [12] used large-area probes and long acoustic pulses to experimentally generate Lamb waves in hot-rolled steel plates up to $16 \mathrm{~mm}$ thickness. They studied the reflection of different Lamb modes at the edges of plates for lamination detection in thick plates. Ditri et al. [13] studied the energy distribution inside a plate for different Lamb wave modes (symmetric and antisymmetric) and tried to predict whether or not modes would be sensitive to a crack of a given depth. Ghosh et al. [14] examined Lamb wave propagation in large plates and its use in internal defect flaw identification. They identified Lamb modes which are most efficient for detecting different types of internal defects. Silva et al. [15] studied hidden corrosion detection in aircraft aluminum structures using a noncontact laser-based ultrasonic technique. They defined the presence of back surface corrosion by noting the loss of the $S_{1}$ mode near its cutoff frequency.

Wilcox et al. [16] developed a method for quickly predicting the rate of spreading of a dispersive wave-packet as it propagates. It is shown that the duration of a wave-packet increases linearly with the propagation distance. Pruell et al. [17] developed an experimental procedure for characterizing fatigue damage in metallic plates using nonlinear guided waves. In this research, they have shown that Lamb waves can be used to quantitatively assess plasticity driven fatigue damage using established higher harmonic generation techniques.

In this study, an area-scan nondestructive method based on Lamb waves was developed which can be used as a complementary method for point-scan methods such as ultrasonic testing. The test-piece surface was partitioned with some same size areas and the damage probability presence of each area was evaluated by defining a damage presence probability index (DPPI) for each area. Areas with the highest DPPI value highlight the most probable locations of damage in the test-piece. The point-scan nondestructive methods can then be used once these areas are found to identify the damage in detail. The approach was validated by predicting the most probable locations of representative damage including through-thickness hole and crack in aluminum plates.

\section{The Area-Scan Nondestructive Method}

When a Lamb wave is incident on damage or structural boundaries, wave scattering occurs. As a result, captured signals can consist of three parts in time domain including the wave directly coming from the actuator, the damage scattered wave, and the reflections from the structure boundaries. The damage scattered waves can be utilized to investigate the

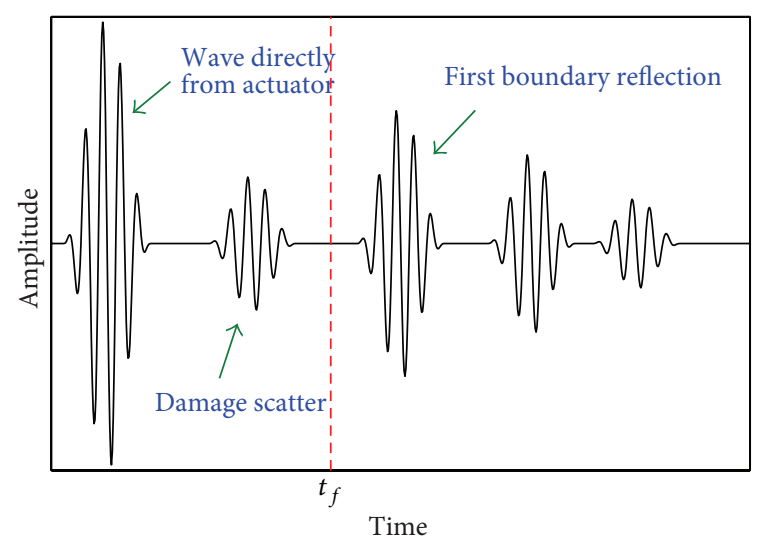

FIgURE 1: A typical Lamb wave signal includes wave that directly comes from actuator, damage scattering, and boundaries reflections.

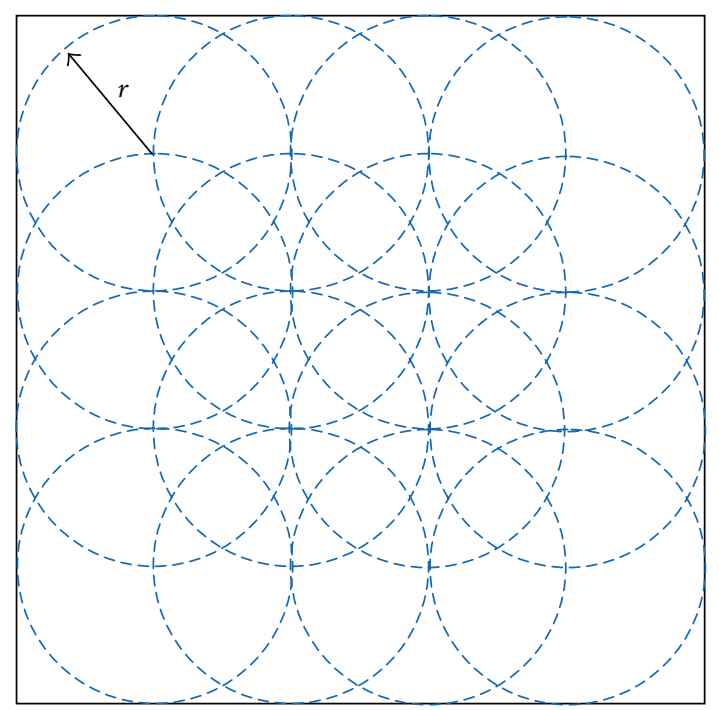

Figure 2: A typical structure partitioned to some same size full circular areas.

damage and usually they are obtained by comparing the current signal with a baseline. However, such a process is affected easily by environmental and operational variability especially when signals are captured via a mobile transducer set. To overcome this difficulty, a baseline-free method is presented in this study. Accordingly, a sensor placed very close to the actuator in the transducer set to capture the wave reflected back from the damage. In this way, the distance between the actuator and the sensor is very short so the wave that directly comes from the actuator only travels a very short time and it appears almost at the very beginning of the captured signal as can be seen in Figure 1. The surface of the structure is partitioned to some same size full circular areas with radius $r$ (areas can cover each other) as can be seen in Figure 2. The size of circular areas depends on the desired accuracy. Choosing the smaller areas results in higher accuracy but increases the time of the NDE process. Since the transducer set can move easily on the structure, it can be placed at the center of such circular areas to examine the damage presence 

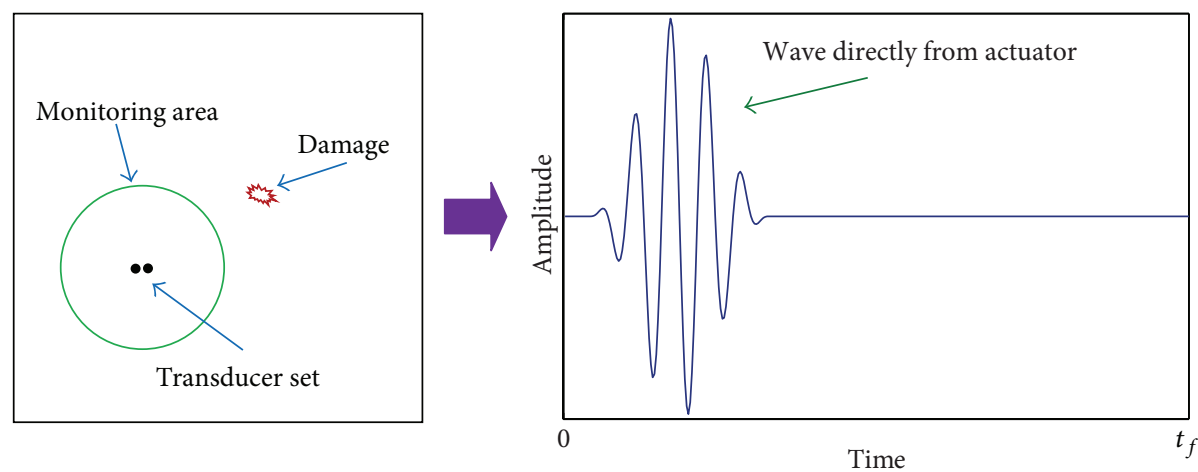

(a)
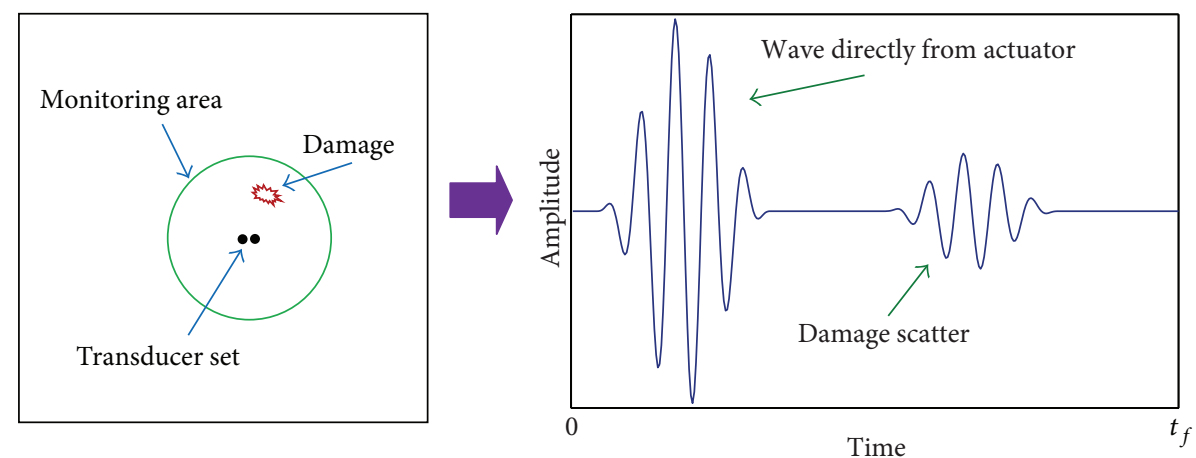

(b)

FIGURE 3: Diagnostic approach: (a) signal captured at monitoring area without damage; (b) signal captured at monitoring area with damage.

probability of each area. To this end, for each area, the current signal for time window $t=\left[0, t_{f}\right]$ (where $t_{f}=2\left(r / c_{g}\right)$ and $c_{g}$ is the selected mode velocity) is collected. Such signals will not include boundaries reflections. Signals captured at areas without damage only include the wave directly coming from the actuator (see Figure 3(a)), while, as can be seen in Figure 3(b), those captured, in areas with damage, in addition to the wave directly coming from the actuator, include the damage scattered waves. Therefore, it is clear that the energy of signals collected in the areas with damage is higher than that acquired in the areas without damage. Since more severe damage results in more and stronger damage scatter, the energy of signals collected in the areas with more severe damage is higher than that acquired in the areas without damage or with tiny damage. Hence, a damage probability presence index (DPPI) can be introduced for each area based on the energy of the current signal captured at that area. Since the root mean square (RMS) of the signal is related to the energy of the signal, the DPPI can be introduced as the RMS of current response as follows:

$$
\begin{aligned}
\operatorname{DPPI}_{i}= & \operatorname{RMS}\left(f_{i}\right) \\
& -\operatorname{Min}\left[\operatorname{RMS}\left(f_{1}\right), \operatorname{RMS}\left(f_{2}\right), \ldots, \operatorname{RMS}\left(f_{N}\right)\right],
\end{aligned}
$$

where $\mathrm{DPPI}_{i}$ is the damage probability presence index of the area $i, N$ is the number of the captured signals, and $\operatorname{RMS}\left(f_{i}\right)$ is the root mean square of the signal $f_{i}$ acquired in the area $i$ and can be computed as follows:

$$
\operatorname{RMS}\left(f_{i}\right)=\sqrt{\frac{\sum_{k=0}^{z} D_{i}\left(k t_{f} / z\right)^{2}}{Z}},
$$

where $D_{i}\left(k t_{f} / z\right)$ is the amplitude of the signal at $t=$ $k t_{f} / z(k=0,1,2, \ldots, z)$ and $Z$ is the number of data points.

As can be seen in Figure 2, some parts near the edges of the structure are not covered with these full circular areas. In order to inspect such parts, the region near the edges of the structure is partitioned with some same size semicircular areas as shown in Figure 4. If the transducer set is placed in the middle of semicircular chord near the structure boundary, the captured signal includes the wave that directly comes from the actuator and the first structure boundary reflection. If any damage exists in these semicircular areas, captured signal in addition to the wave that directly comes from the actuator and the first structure boundary reflection will include the damage scattered waves. Therefore, the energy of the signals collected in the areas with damage is higher than that acquired in the areas without damage. Like the full circular areas, for each semicircular area, a DPPI can be defined using (1). It should be noticed that since the capture signal in the semicircular areas is different from those acquired in the full circular areas, the DPPI of such areas must be compared with each other to highlight the most probable location of the damage near the structure boundaries. 


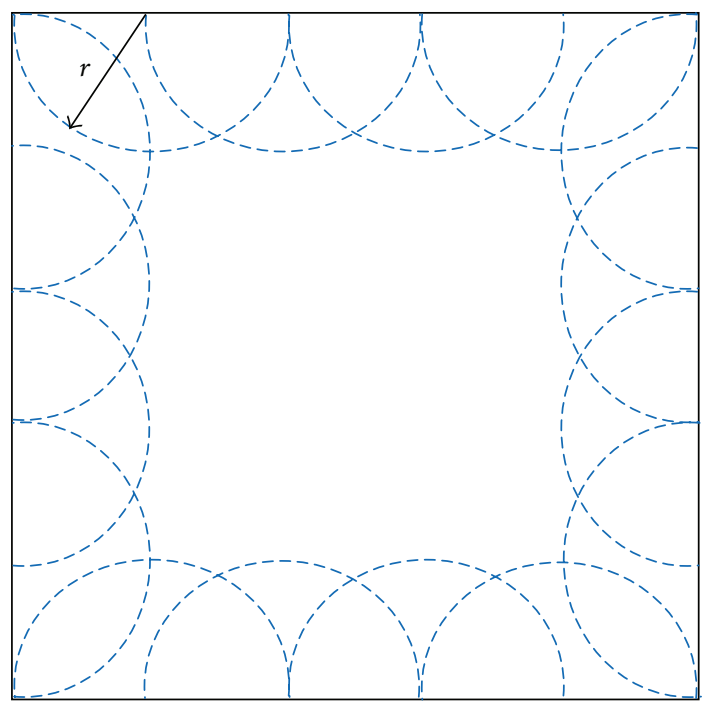

Figure 4: A typical structure partitioned to some same size semicircular areas.

TABLE 1: Material properties of PZT transducer.

\begin{tabular}{lc}
\hline Product name & APC 851 \\
\hline Geometry $(\mathrm{mm})$ & $\emptyset=6.6, h_{\text {PZT }}=0.24$ \\
Density $\left(\mathrm{g} / \mathrm{cm}^{3}\right)$ & 7.6 \\
Electromechanical coupling factor $\left(k_{p}\right)$ & 0.71 \\
Voltage constant $g_{33}(\mathrm{Vm} / \mathrm{N})$ & $24.8 \times 10^{-3}$ \\
Charge constant $d_{33}(\mathrm{~m} / \mathrm{V})$ & $400 \times 10^{-12}$ \\
Relative dielectric constant $K^{T}$ & 1950 \\
Frequency constant $N_{T}(\mathrm{~Hz} \cdot \mathrm{m})$ & 2040 \\
Elastic constant $E(\mathrm{GPa})$ & 63 \\
\hline
\end{tabular}

When the areas with the highest DPPI values are defined, the point-scan nondestructive techniques will be used to identify the damage in detail. This process starts from the area with the maximum DPPI value. Once the damage of this area is quantified, the NDE engineer based on the severity of the defined damage and also the application of the structure can decide if it is necessary to monitor other areas with lower DPPI values or not.

\section{Experimental Setup}

In order to generate and collect Lamb waves in the structure, a mobile handmade transducer set is designed. To design such a transducer set, its flexibility must be taken into consideration, because it should be able to easily move on the structure to excite and collect Lamb waves at different locations of the structure. In the transducer set, a sensor is collocated with the actuator to capture the wave reflected back from the damage. Piezoelectric discs (APC 851) are utilized in the transducer set whose diameter and thickness are $6.6 \mathrm{~mm}$ and $0.24 \mathrm{~mm}$, respectively. The piezoelectric discs properties are given in Table 1 . The PZT sensor and the actuator are attached to the bottom of the separate holders. Two screws passing through a top square plate maintain the holders in balance.

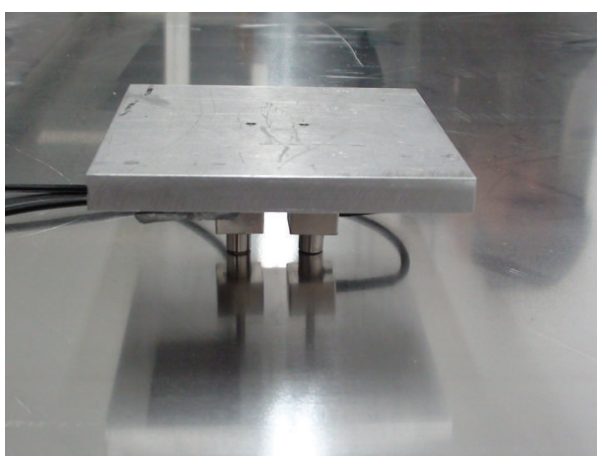

Figure 5: The designed handmade transducer set.

Figure 5 shows the designed transducer set. To facilitate the signal separation process, only the $A_{0}$ mode of Lamb wave is generated in the structure. To achieve this, a kind of grease lubricant is applied under the bottom surface of the actuator. In this way, strong vertical pressure will be generated by the actuator to the plate to excite the $A_{0}$ mode and decline shear force between the actuator and the plate to weaken the $S_{0}$ mode. By employing the above technique, a pure $A_{0}$ mode can be excited.

A 5-cycle sinusoidal toneburst enclosed in a Hanning window at a central frequency of $50 \mathrm{kHz}$ was generated using an arbitrary waveform generation unit (Agilent 33220A). The analogue signal was amplified to $85(\mathrm{~V})$ using a linear amplifier (T\&C power conversion, Inc., AG series) to drive each PZT actuator in turn. Wave signals were captured using an oscilloscope (Agilent DSO5032A) at a sampling rate of $10 \mathrm{MHz}$. Sampled signals were transmitted into the central processing unit for further analysis. The $A_{0}$ mode velocity is assumed to be constant in this research.

\section{Applications}

In this section, two selected experimental studies are presented that demonstrate the capability of the developed method to detect the most probable locations of damage in structures.

An aluminum plate $(1000 \mathrm{~mm} \times 1000 \mathrm{~mm} \times 3 \mathrm{~mm})$ was fixed along its four edges on a testing table. A throughthickness hole $(\emptyset=2 \mathrm{~cm})$ was introduced into the plate, as seen in Figure 6. The surface of the structure was partitioned to some same size full circular areas with radius $20 \mathrm{~cm}$ as shown in Figure 2. The transducer set was placed at the center of such areas (areas: A1, A2,.., A16 shown in Figure 6) to generate and collect Lamb waves in each area. The signal acquisition locations are listed in Table 2 . The $A_{0}$ velocity at $50 \mathrm{KHz}$ in the aluminum plate experimentally was defined $(V=1955.7 \mathrm{~m} / \mathrm{s})$. Therefore, at each area, the current signal for time window $t=[0,204.5] \mu$ s was collected. All the captured signals were normalized to compensate the performance difference among transducer set at different locations. Typical responses collected by the transducer set at the center of areas A8 and A10 are shown in Figure 7. The DPPI of different areas are calculated using (1) and shown in 


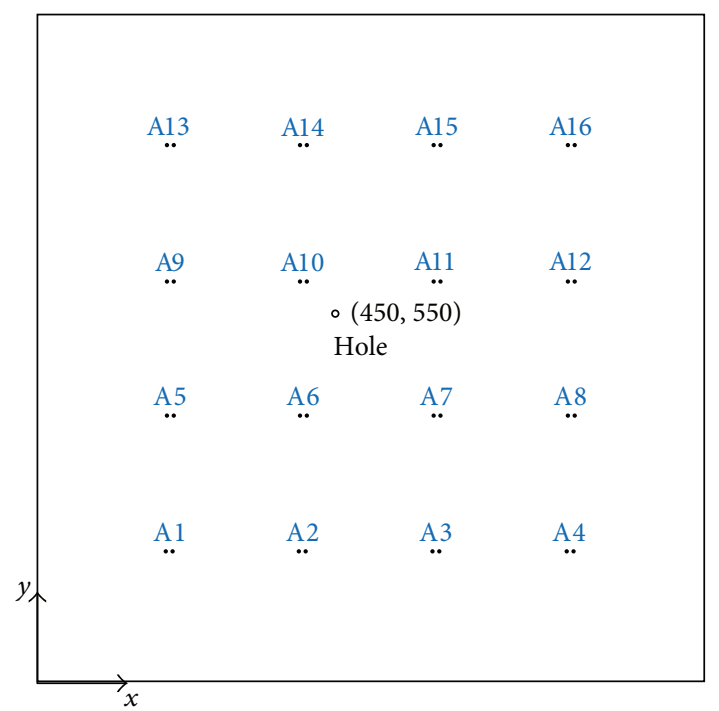

FIGURE 6: Signal acquisition locations for identifying a throughthickness hole in aluminum plate (unit: $\mathrm{mm}$ ).

TABLE 2: Coordinates of signal acquisition locations on aluminum plate.

\begin{tabular}{lccc}
\hline Area & $\begin{array}{c}\text { Coordinates } \\
(x, y) /(\mathrm{mm})\end{array}$ & Location & $\begin{array}{c}\text { Coordinates } \\
(x, y) /(\mathrm{mm})\end{array}$ \\
\hline A1 & $(200,200)$ & A9 & $(200,600)$ \\
A2 & $(400,200)$ & A10 & $(400,600)$ \\
A3 & $(600,200)$ & A11 & $(600,600)$ \\
A4 & $(800,200)$ & A12 & $(800,600)$ \\
A5 & $(200,400)$ & A13 & $(200,800)$ \\
A6 & $(400,400)$ & A14 & $(400,800)$ \\
A7 & $(600,400)$ & A15 & $(600,800)$ \\
A8 & $(800,400)$ & A16 & $(800,800)$ \\
\hline
\end{tabular}

Figure 8. As can be seen in Figure 8, the maximum value of DPPI is defined when the transducer set is placed at the center of the area A10. Therefore, as can be seen in Figure 9, the most probable location of damage is area A10 which includes the real hole and shows the effectiveness of the presented method in highlighting the most probable location of damage in structures. Since the damage scattered waves can be also sensed by the signals collected at areas A6 and A11, the DPPI values of these areas are more than the other areas in which the captured signal at those areas does not include the damage scattered waves. Nevertheless, because of lower linear distance between the damage and the center of area A10 compared with the center of areas A6 and A11, the DPPI value of the area A10 is more than the DPPI values of the areas A6 and A11.

As an extension of the above application, the approach was used to identify through-thickness crack $(25 \mathrm{~mm}$ long and $1.5 \mathrm{~mm}$ wide) in an aluminum plate with the same dimensions as in previous study, as seen in Figure 10. The same process was performed to identify the most probable location of damage. Figure 11 shows the DPPI of different full circular

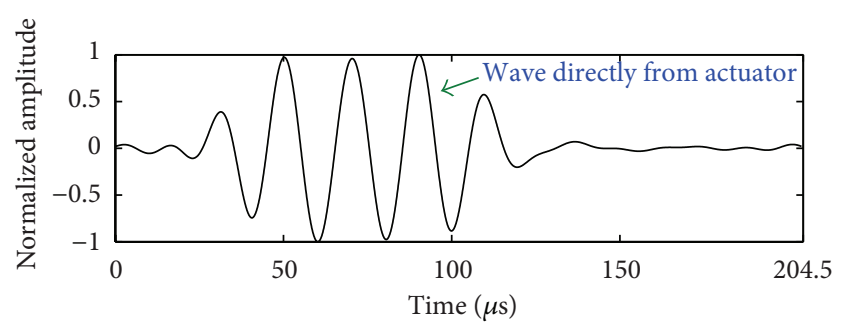

(a)

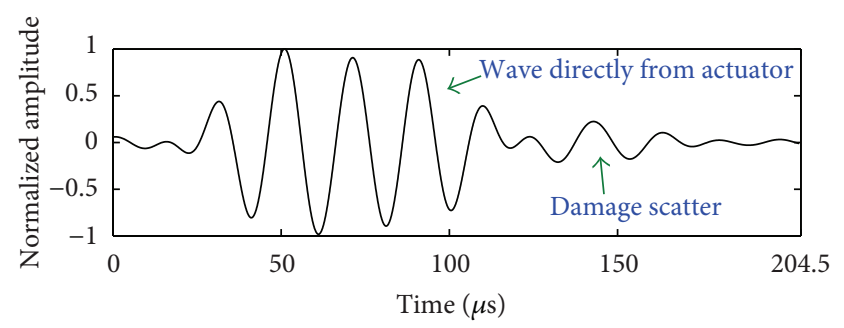

(b)

FIGURE 7: Typical Lamb wave signals collected on aluminum plate with a through-thickness hole: at (a) A8 and (b) A10.

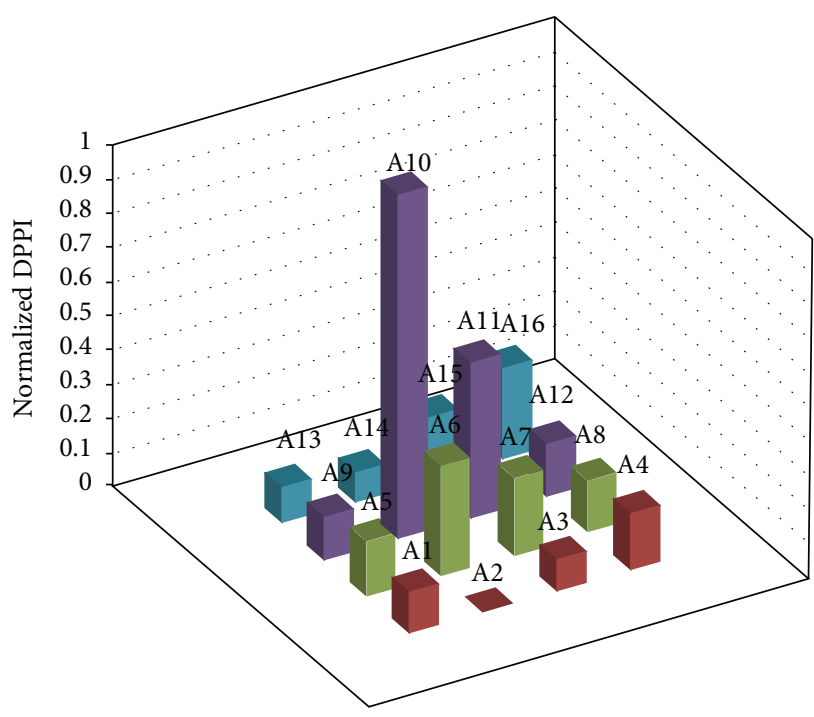

FIGURE 8: Damage probability presence index (DPPI) values defined at different areas of aluminum plate with through-thickness hole.

areas of the aluminum plate with through-thickness crack. As can be recognized from Figure 11, the maximum value of DPPI is defined when the transducer set placed at the center of the area A3. Therefore as can be seen in Figure 12 the most probable location of damage is the area A3 which includes the real through-thickness crack and again demonstrates the effectiveness of presented method in highlighting the most probable location of the damage in the structures.

It is also worthy to be mentioned that although the incident of Lamb waves on the interior damage such as delamination results in scatter with lower amplitude compared with the surface damage, still the energy of signals collected in areas with the interior damage is higher than in the areas 


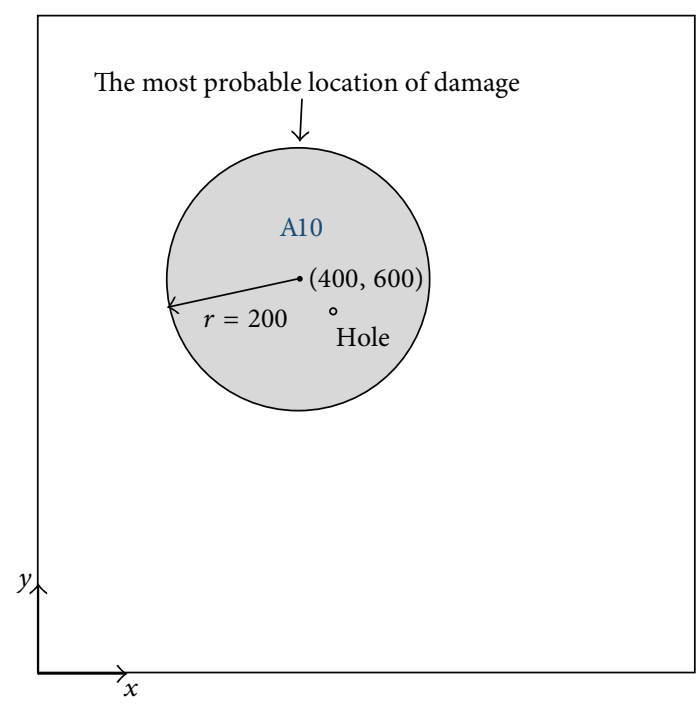

Figure 9: The most probable location of damage in aluminum plate with through-thickness hole.

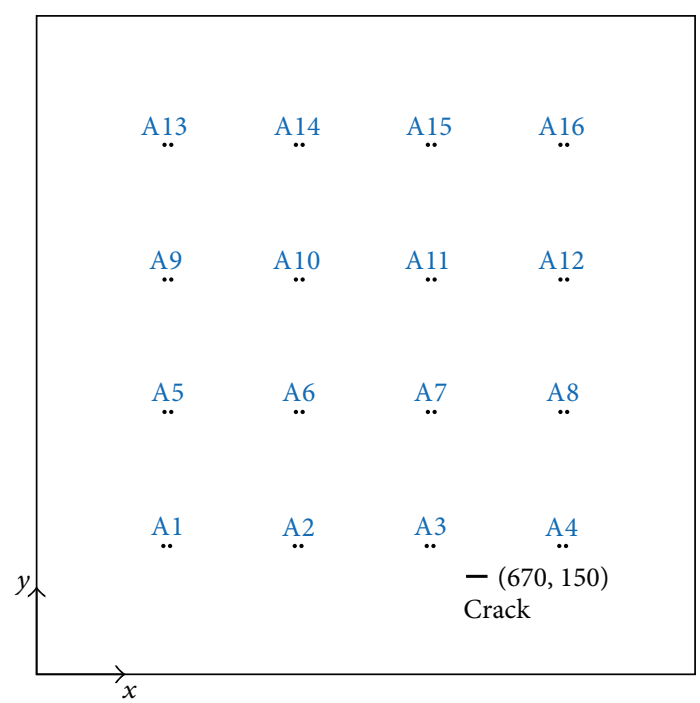

FIGURE 10: Signal acquisition locations for identifying a throughthickness crack in aluminum plate (unit: $\mathrm{mm}$ ).

without damage. As a result, such damage can also be detected by using the developed technique.

\section{Conclusion}

In this study, an area-scan nondestructive method based on Lamb waves was developed to identify the most probable locations of damage prior to point-scan test. The test-piece surface was partitioned with some smaller areas and the damage probability presence of each area was evaluated. To this end, the $A_{0}$ mode of Lamb wave was generated and collected at each area using a mobile handmade transducer set. Subsequently, a damage presence probability index (DPPI) based on the energy of the captured responses was

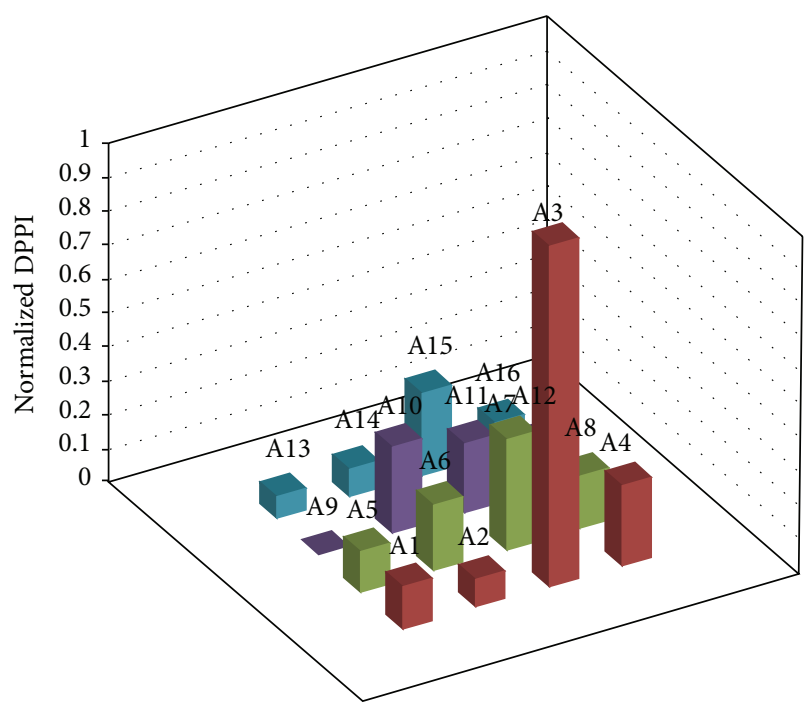

FIGURE 11: Damage probability presence index (DPPI) values defined at different areas of aluminum plate with through-thickness crack.

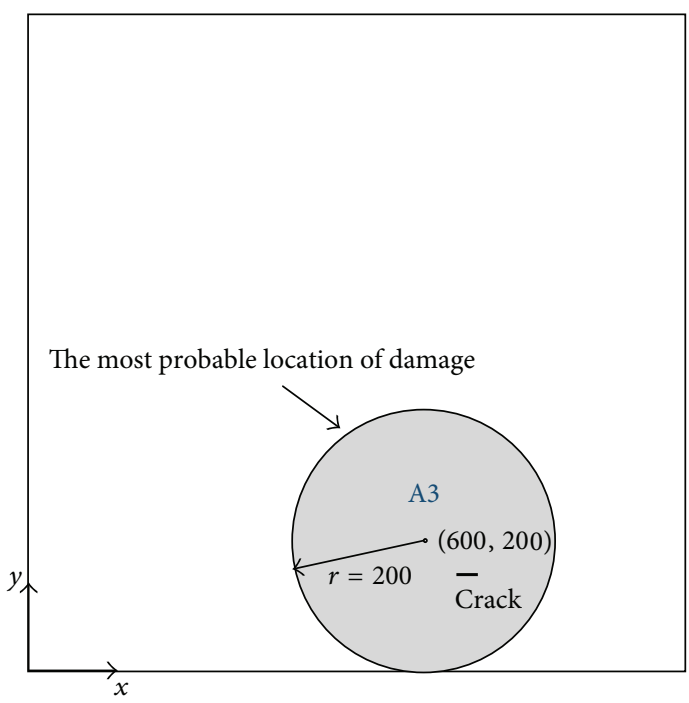

FIGURE 12: The most probable location of damage in aluminum plate with through-thickness crack.

defined for each area. Areas with the highest DPPI values highlight the most probable location of damage in test-piece. Conventional point-scan NDE methods, such as ultrasonic testing, can then be used to characterize the damage in detail. Satisfactory results from two selected damage cases, including through-thickness hole and crack in structural plates, have demonstrated the effectiveness of the developed method in defining the most probable location of damage in the structures which qualifies the presented method as an appropriate complement for other conventional point-scan NDE methods and in this way decreases the time and cost of the nondestructive evaluation. 


\section{Conflict of Interests}

The authors declare that there is no conflict of interests regarding the publication of this paper.

\section{Acknowledgments}

This work was supported by the National Natural Science Foundation of China (11172053 and 91016024) and the New Century Excellent Talents in University (NCET-11-0055) and the Fundamental Research Funds for the Central Universities (DUT12LK33).

\section{References}

[1] Y. Bar-Cohen, "Emerging NDE technologies and challenges at the beginning of the 3rd millennium-part II," Journal of Nondestructive Testing, vol. 5, no. 2, pp. 1-10, 2000, http://ndt.net/ article/v05n02/barcohen/barcohen.htm.

[2] J. L. Rose, Ultrasonic Waves in Solid Media, CUP, Cambridge, UK, 1999.

[3] N. Guo and P. Cawley, "Lamb waves for the NDE of composite laminates," in Review of Progress in Quantitative Nondestructive Evaluation, vol. 11, pp. 1443-1450, Springer US, 1992.

[4] H. Lamb, "On waves in an elastic plate," Proceedings of the Royal Society of London, vol. 93, pp. 114-128, 1917.

[5] F. A. Firestone and D. S. Ling, "Methods and means of generating and utilizing vibration of waves in plates," US patent no. 2536128, 1951.

[6] D. N. Alleyne and P. Cawley, "The interaction of Lamb waves with defects," IEEE Transactions on Ultrasonics, Ferroelectrics, and Frequency Control, vol. 39, no. 3, pp. 381-397, 1992.

[7] F. Levent Degertekin and B. T. Khuri-Yakub, "Lamb wave excitation by hertzian contacts with applications in NDE," IEEE Transactions on Ultrasonics, Ferroelectrics, and Frequency Control, vol. 44, no. 4, pp. 769-779, 1997.

[8] A. Oishi, K. Yamada, S. Yoshimura, G. Yagawa, S. Nagai, and Y. Matsuda, "Neural network-based inverse analysis for defect identification with laser ultrasonics," Research in Nondestructive Evaluation, vol. 13, no. 2, pp. 79-95, 2001.

[9] E. K. Dimitriadis, C. R. Fuller, and C. A. Rogers, "Piezoelectric actuators for distributed vibration excitation of thin plates," Journal of Vibration and Acoustics, vol. 113, pp. 100-107, 1991.

[10] R. S. C. Monkhouse, P. W. Wilcox, M. J. S. Lowe, R. P. Dalton, and P. Cawley, "Rapid monitoring of structures using interdigital Lamb wave transducers," Smart Materials and Structures, vol. 9, no. 3, pp. 304-309, 2000.

[11] D. C. Worlton, "Ultrasonic testing with Lamb waves," Nondestructive Testing, vol. 15, no. 4, pp. 218-222, 1957.

[12] E. Lehfeldt and P. Höller, "Lamb waves and lamination detection," Ultrasonics, vol. 5, no. 4, pp. 255-257, 1967.

[13] J. J. Ditri, J. L. Rose, and G. Chen, "Mode selection criteria for defect detection optimization using Lamb waves," Review of Progress in Quantitative Nondestructive Evaluation, vol. 11, pp. 2109-2115, 1991.

[14] T. Ghosh, T. Kundu, and P. Karpur, "Efficient use of Lamb modes for detecting defects in large plates," Ultrasonics, vol. 36, no. 7, pp. 791-801, 1998.
[15] M. Z. Silva, R. Gouyon, and F. Lepoutre, "Hidden corrosion detection in aircraft aluminum structures using laser ultrasonics and wavelet transform signal analysis," Ultrasonics, vol. 41, no. 4, pp. 301-305, 2003.

[16] P. Wilcox, M. Lowe, and P. Cawley, "Effect of dispersion on longrange inspection using ultrasonic guided waves," NDT and E International, vol. 34, no. 1, pp. 1-9, 2001.

[17] C. Pruell, J.-Y. Kim, J. Qu, and L. J. Jacobs, "Evaluation of fatigue damage using nonlinear guided waves," Smart Materials and Structures, vol. 18, no. 3, Article ID 035003, 2009. 

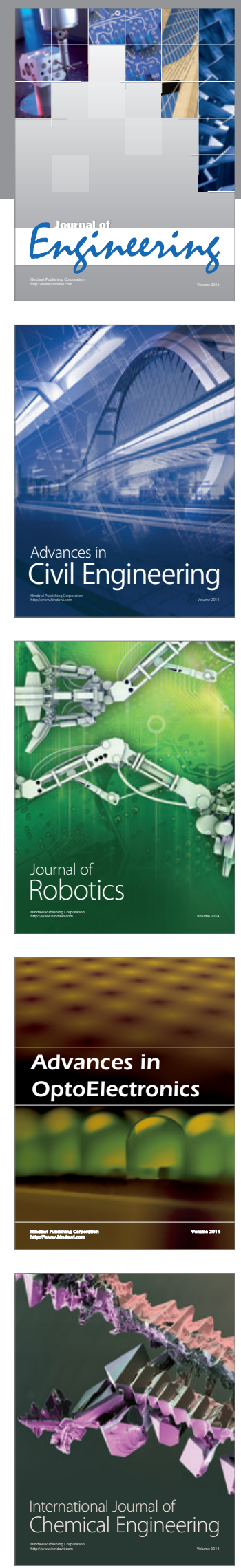

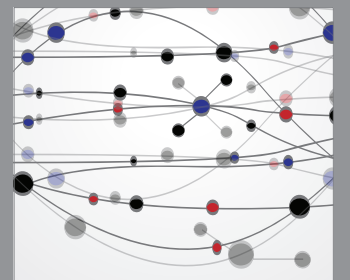

The Scientific World Journal
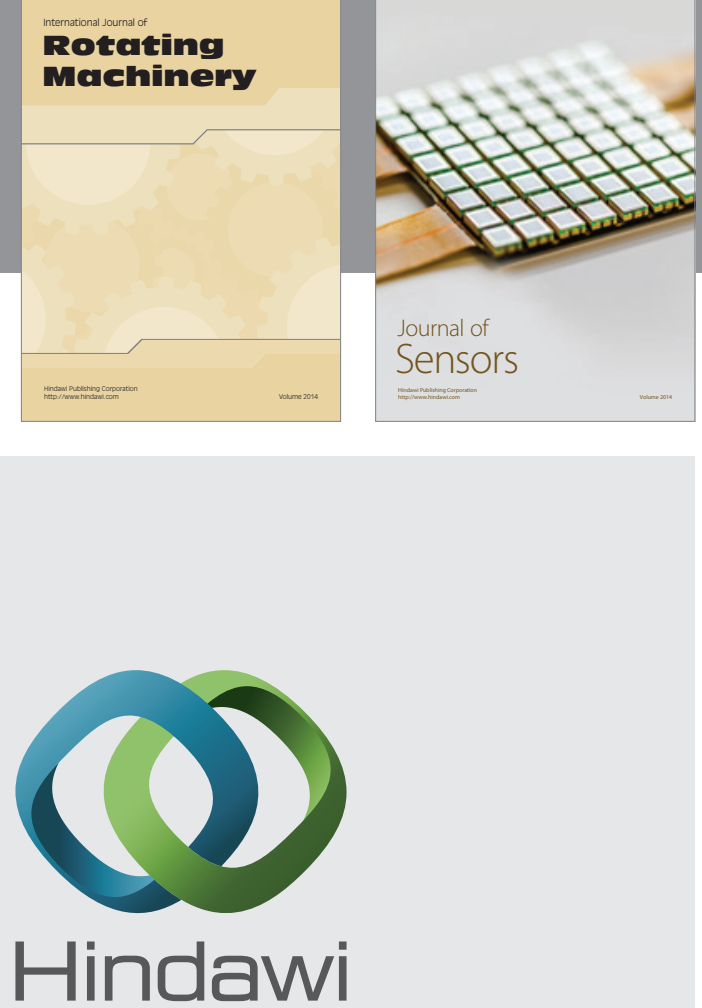

Submit your manuscripts at http://www.hindawi.com
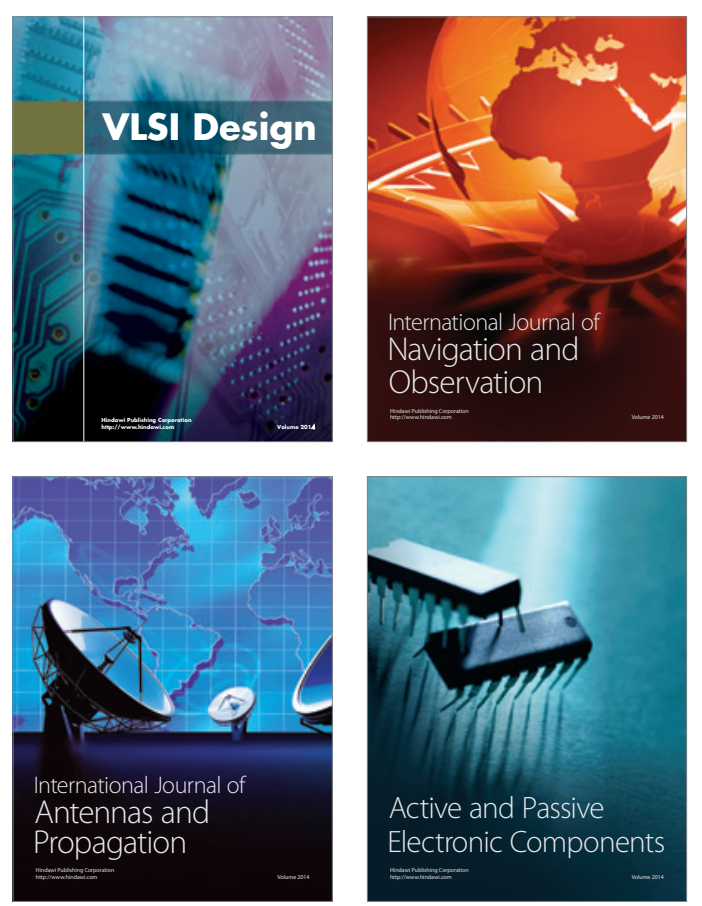
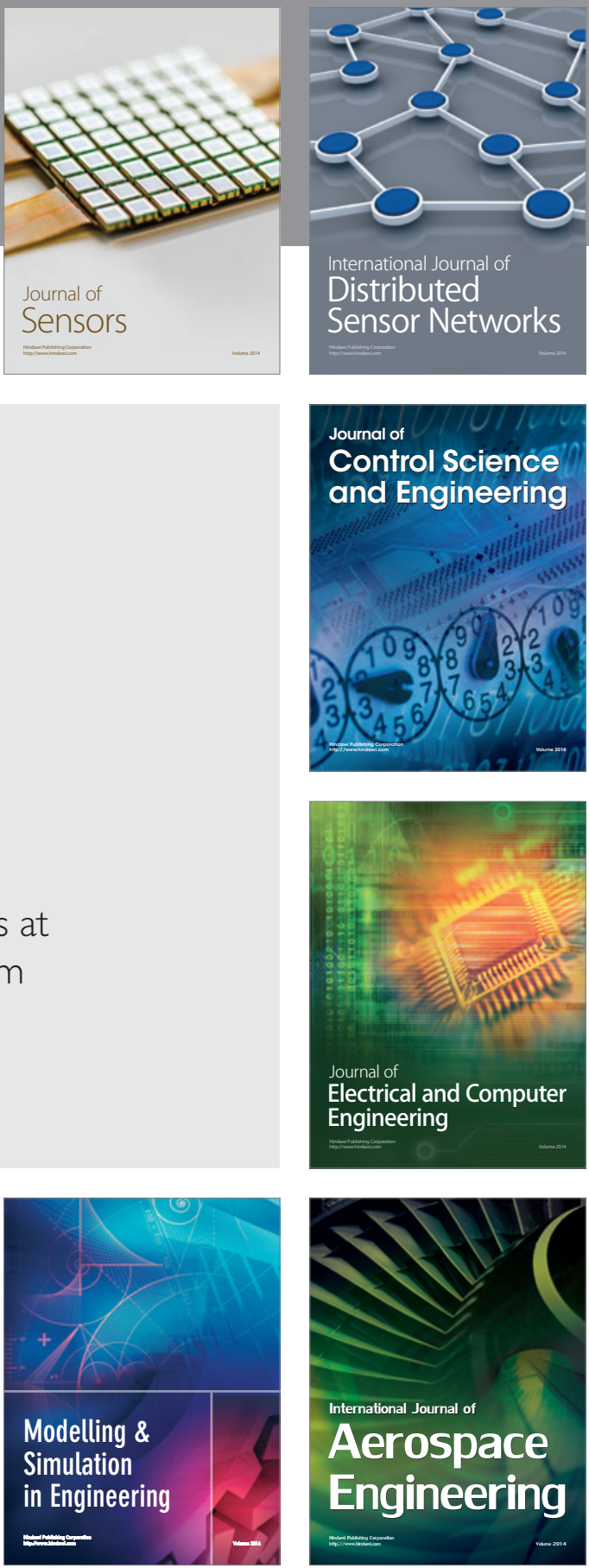

Journal of

Control Science

and Engineering
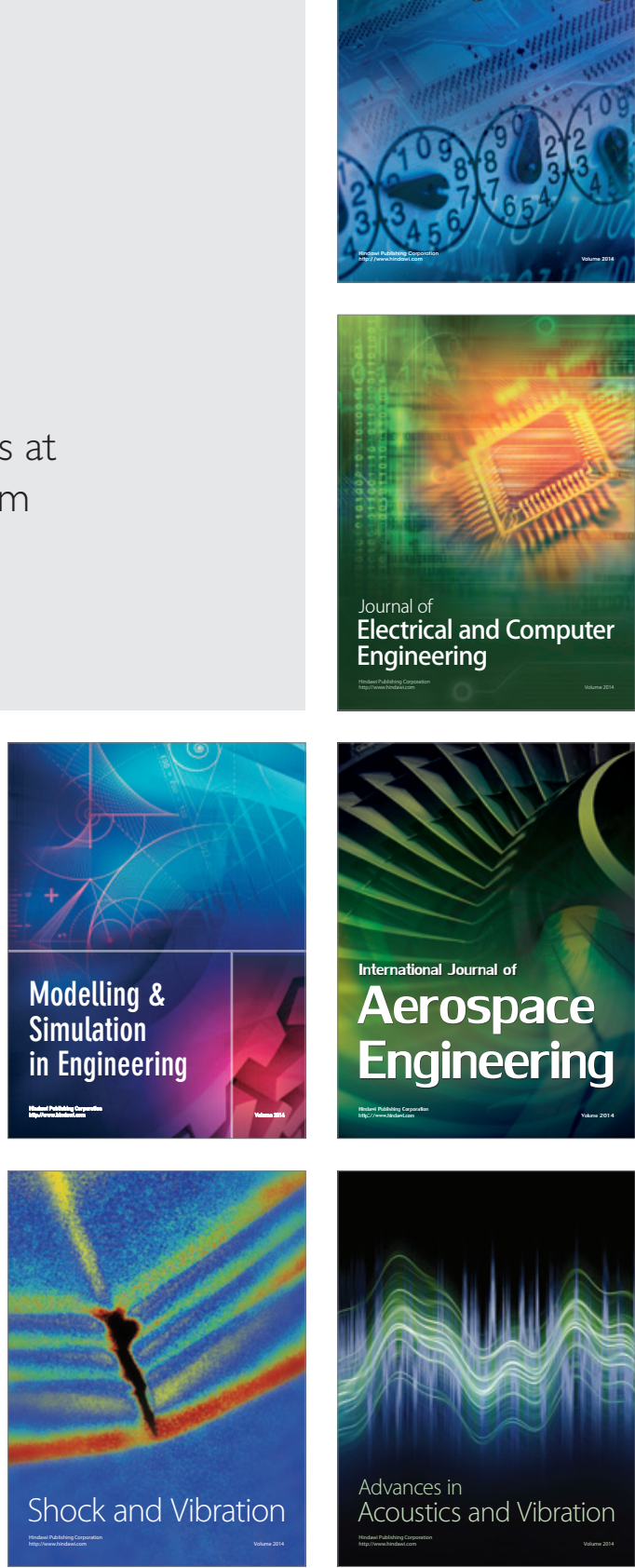